\section{Long-term results of half-dose photodynamic therapy for chronic central serous chorioretinopathy with contrast sensitivity changes}

SH Karakus'1, B Basarir', EY Pinarci², EU Kirandi and A Demirok'
${ }^{1}$ Department of Ophthalmology, Beyoglu Eye Training and Research Hospital, Istanbul, Turkey

${ }^{2}$ Department of Ophthalmology, Faculty of Medicine, Baskent University, Istanbul, Turkey

Correspondence: SH Karakus, Department of Ophthalmology, Beyoglu Eye Training and Research Hospital, Bereketzade cami sok. No:2/1 Kuledibi, Beyoglu, Istanbul 34100, Turkey.

Tel: +90 21225159 00; Fax: +90 21227761 11; Cell phone:

+90 53322376 44;

E-mail: karakussezen@ hotmail.com

Received: 3 August 2012 Accepted in revised form: 22 January 2013

Published online: 22 March 2013

\begin{abstract}
Purpose To evaluate the efficacy and safety of photodynamic therapy (PDT) with halfdose verteporfin in patients with chronic central serous chorioretinopathy (CCSC) and retinal functional changes, by functional acuity contrast test (FACT).

Methods In this study, 27 eyes of 24 patients with CCSC were treated with PDT with halfdose verteporfin. Best-corrected visual acuity (BCVA), central foveal thickness (CFT) and resolution of subretinal fluid on optical coherence tomography (OCT), and leakage on fluorescein angiography (FA) and indocyanine green angiography (ICGA) were assessed. Contrast sensitivity test was performed at baseline and at 12th month for investigating retinal functional changes. Results The mean follow-up period was $25.33 \pm 11.08$ months. The mean age was $43.7 \pm 8.6$ years. Seventeen patients were male (70.8\%) and seven patients were female (29.2\%). Post PDT at 1st, 3rd, 6th, 12th month and at last follow-up, BCVA were significantly improved compared with the baseline BCVA $(P<0.001)$, and CFT post PDT were significantly thinner than the baseline measurement $(P<0.001)$. There was significant difference between pre- and postPDT 12th month contrast sensitivities at all five different spatial frequency channels $(P<0.01)$

Conclusions Half-dose PDT is an effective and safe method in the treatment of CCSC with anatomical and functional success. The measurement of contrast sensitivity by FACT
\end{abstract}

can be useful for evaluating the functional effectiveness of half-dose PDT for CCSC. Eye (2013) 27, 612-620; doi:10.1038/eye.2013.24; published online 22 March 2013

Keywords: central serous chorioretinopathy; photodynamic therapy; verteporfin; contrast sensitivity; functional acuity contrast test (FACT)

\section{Introduction}

Central serous chorioretinopathy (CSC) is characterized by serous detachment of the neurosensory retina at the posterior pole. ${ }^{1}$ It is commonly a self-limiting disease and usually resolves spontaneously within 3 or 4 months. ${ }^{2}$ However, some patients experience a persistent serous detachment for at least 6 months, or so-called CCSC. ${ }^{3-5}$ The pathogenesis of CSC is now believed to be related to abnormal choroidal circulation, as demonstrated by ICGA. ${ }^{6-9}$ Congested and dilated choroidal vessels, choroidal staining, and hyperfluorescence have been shown in the middle and late phases of ICGA. ${ }^{10}$

In recent years, PDT has been reported to be an effective treatment option for CSC in most patients. ${ }^{11-14}$ The therapeutic mechanism of PDT is postulated to be caused by short-term choriocapillaris hypoperfusion and long-term choroidal vascular remodeling, which reduces choroidal congestion, vascular hyperpermeability, and extravascular leakage. ${ }^{15}$ However, conventional PDT in CSC can result in potential complications such as retinal pigment 
epithelium (RPE) atrophy, choroidal ischemia, and secondary choroidal neovascularization $(\mathrm{CNV}) .{ }^{12,15,16}$ By reducing the dose of verteporfin or shortening the time of laser emission, it has been previously demonstrated that the potential adverse effects may be minimized while resulting in good treatment efficacy in the treatment of chronic CSC. ${ }^{17-20}$

The purpose of this article was to report the outcomes of half-dose PDT in patients with CCSC. The distinctive mark of this article is evaluating retinal functional changes before and after the treatment by performing functional acuity contrast test (FACT).

\section{Materials and methods}

\section{Study design and patient selection}

We prospectively studied 27 eyes of 24 patients with symptomatic chronic CSC for at least 6 months. Some of the inclusion criteria were persistence of subretinal fluid (SRF) and/or pigment epithelial detachment (PED) for at least 6 months with subjective symptoms. Patients who received any previous treatment, including PDT or focal thermal laser photocoagulation for CSC, or who had FA or ICGA findings of CNV, polyploidal choroidal vasculopathy (PCV), or other maculopathy on clinical examination were excluded. Patients with posterior cystoid retinal degeneration (PCRD), which is considered a negative prognostic factor, were also excluded. ${ }^{21}$

The subgroup analysis of duration of symptoms, presence of PED, and age was carried out to evaluate the prognostic effect of these conditions. Patients were evaluated in two groups according to the duration of symptoms: 'duration of 6-9 months' and '>9 months'. The eyes with combined SRF and PED were compared with the eyes with only SRF. Moreover, the patients were divided into two groups: ' $<45$ years' and ' $\geq 45$ years' for evaluating the age factor.

Informed consent was obtained from all patients before the procedure, and the study protocol was approved by an institutional review board. We certify that all applicable institutional and governmental regulations concerning the ethical use of human volunteers were followed during this research.

\section{Photodynamic therapy}

PDT was performed using half the normal dose of verteporfin $\left(3 \mathrm{mg} / \mathrm{m}^{2}\right)$. Verteporfin was infused over $10 \mathrm{~min}$, followed by delivery of laser at $689 \mathrm{~nm}$ at $15 \mathrm{~min}$ from the commencement of infusion to target the area of choroidal dilation and hyperpermeability in ICGA. A total light energy of $50 \mathrm{~J} / \mathrm{cm}^{2}$ over $83 \mathrm{~s}$ was delivered to the area of choroidal hyperperfusion. To avoid overtreatment of the choroidal vasculature and causing choroidal ischemia, the laser spot size was set maximum of $4500 \mu \mathrm{m} .{ }^{18}$ In one case, CCSC was observed in both eyes at the same time, just after completing the first eye's treatment, the fellow eye's treatment was initiated by using the same duration and energy. Other two bilateral cases were treated in different sessions. After treatment, all patients were given protective spectacles and were instructed to avoid strong light for 3 days.

\section{Baseline and follow-up examinations}

Patients were assessed at baseline and followed up at 1st, 3rd, 6th, 12th month, and every 6 months until the last visit after PDT. At the baseline and all post-PDT visits, Snellen BCVA, fundus examination, and macular OCT were performed in all patients. The OCT recordings were performed using an OCT 3 (Stratus OCT, Carl Zeiss Meditec, Inc., Dublin, CA, USA) for measurement of CFT. Both vertical and horizontal scans of $6.0 \mathrm{~mm}$ centered on the fovea were obtained. CFT was measured manually using the retinal thickness mode with calipers and is defined as the distance between the inner surface of the retinal pigment epithelium and the outer surface of the neurosensory retina at the fovea. FA and ICGA were performed at baseline and 3 months after PDT.

Functional acuity contrast test (FACT, Stereo Optical Co., Chicago, IL, USA) using the OPTEC $6500 \mathrm{P}$ vision testing system in all patients with best correction under photopic and mesopic conditions without glare, and mesopic condition with glare at baseline and 12 months after PDT was performed for assessment of retinal function. The FACT sine-wave grating chart tests five spatial frequencies (A: 1.5 cycle per degree (cpd), B: 3 cpd, C: 6 cpd, D: 12 cpd, and $\mathrm{E}: 18 \mathrm{cpd}$ ) and nine levels of contrast. The patient determines the last grating seen for each row (A, B, C, D, and $\mathrm{E}$ ) and reports the orientation of the grating: right, up, or left. The last correct grating seen for each spatial frequency is plotted on a contrast sensitivity curve. ${ }^{22}$

\section{Statistical analysis}

Statistical analysis were performed using SPSS version 16.0 (SPSS, Inc., Chicago, IL, USA). Snellen BCVA was converted to logarithm of the minimum angle of resolution (logMAR) BCVA and its corresponding line number for analysis. Paired $t$-test or Wilcoxon's rank test was used for comparison of variables before and after the treatment. $P \leq 0.05$ was considered statistically significant.

\section{Results}

Twenty-seven eyes of 24 patients diagnosed with CCSC were chosen and treated with half-dose verteporfin PDT 
Table 1 Data of patients diagnosed with chronic central serous chorioretinopathy (CCSC)

\begin{tabular}{|c|c|c|c|c|c|c|c|c|c|c|}
\hline $\begin{array}{l}\text { Patient } \\
\text { no }\end{array}$ & $\begin{array}{c}\text { Age } \\
\text { (years) }\end{array}$ & Sex & $R$ vs $L$ & $\begin{array}{c}\text { Duration of } \\
\text { symptoms } \\
\text { (months) }\end{array}$ & PED & $\begin{array}{c}\text { Baseline } \\
\text { BCVA }\end{array}$ & $\begin{array}{l}\text { Baseline } \\
\text { CFT }(\mu m)\end{array}$ & $\begin{array}{l}\text { Final } \\
\text { BCVA }\end{array}$ & $\begin{array}{l}\text { Final } \\
\text { CFT } \\
(\mu m)\end{array}$ & $\begin{array}{r}\text { Follow-up } \\
\text { (months) }\end{array}$ \\
\hline 1 & 51 & $\mathrm{M}$ & $\mathrm{R}$ & 24 & Yes & 0.016 & 384 & 0.1 & 160 & 36 \\
\hline 2 & 49 & $\mathrm{M}$ & $\mathrm{R}$ & 15 & No & 0.5 & 236 & 0.7 & 131 & 42 \\
\hline 3 & 49 & $\mathrm{M}$ & $\mathrm{L}$ & 6 & Yes & 0.9 & 255 & 1.0 & 152 & 36 \\
\hline \multirow[t]{2}{*}{4} & 43 & $\mathrm{~F}$ & $\mathrm{R}$ & 12 & Yes & 0.9 & 231 & 1.0 & 181 & 36 \\
\hline & 43 & $\mathrm{~F}$ & L & 6 & No & 0.9 & 294 & 1.0 & 160 & 36 \\
\hline 5 & 27 & $\mathrm{~F}$ & $\mathrm{R}$ & 18 & Yes & 0.3 & 210 & 1.0 & 189 & 36 \\
\hline 6 & 51 & $\mathrm{M}$ & L & 18 & Yes & 0.8 & 209 & 1.0 & 171 & 36 \\
\hline 7 & 61 & $\mathrm{M}$ & L & 15 & No & 1.0 & 226 & 1.0 & 168 & 36 \\
\hline 8 & 39 & $\mathrm{~F}$ & $\mathrm{R}$ & 15 & Yes & 0.3 & 288 & 0.4 & 146 & 36 \\
\hline 9 & 34 & $\mathrm{M}$ & $\mathrm{R}$ & 9 & No & 0.5 & 494 & 1.0 & 190 & 36 \\
\hline 10 & 31 & $\mathrm{M}$ & $\mathrm{L}$ & 12 & No & 0.9 & 370 & 1.0 & 150 & 36 \\
\hline 11 & 39 & $\mathrm{M}$ & $\mathrm{R}$ & 12 & Yes & 1.0 & 246 & 1.0 & 207 & 36 \\
\hline 12 & 32 & $\mathrm{~F}$ & $\mathrm{~L}$ & 7 & No & 0.8 & 285 & 1.0 & 163 & 30 \\
\hline 13 & 51 & $\mathrm{~F}$ & $\mathrm{R}$ & 6 & No & 0.9 & 525 & 1.0 & 190 & 24 \\
\hline 14 & 44 & $\mathrm{M}$ & $\mathrm{L}$ & 24 & No & 1.0 & 422 & 1.0 & 176 & 24 \\
\hline 15 & 58 & $\mathrm{~F}$ & $\mathrm{~L}$ & 18 & No & 0.4 & 356 & 0.6 & 132 & 12 \\
\hline 16 & 59 & $\mathrm{M}$ & $\mathrm{R}$ & 8 & No & 0.8 & 370 & 1.0 & 169 & 18 \\
\hline 17 & 45 & $\mathrm{M}$ & $\mathrm{L}$ & 6 & No & 0.8 & 448 & 1.0 & 182 & 18 \\
\hline \multirow[t]{2}{*}{18} & 42 & $\mathrm{M}$ & $\mathrm{R}$ & 18 & No & 0.8 & 308 & 1.0 & 172 & 18 \\
\hline & 42 & $\mathrm{M}$ & $\mathrm{L}$ & 8 & Yes & 1.0 & 270 & 1.0 & 197 & 18 \\
\hline 19 & 46 & $\mathrm{M}$ & $\mathrm{L}$ & 18 & No & 0.8 & 241 & 0.8 & 130 & 12 \\
\hline 20 & 44 & $\mathrm{M}$ & $\mathrm{L}$ & 9 & No & 1.0 & 389 & 1.0 & 168 & 12 \\
\hline \multirow[t]{2}{*}{21} & 42 & $\mathrm{M}$ & $\mathrm{R}$ & 6 & No & 0.6 & 435 & 1.0 & 174 & 12 \\
\hline & 42 & M & $\mathrm{L}$ & 12 & No & 0.4 & 196 & 0.8 & 154 & 12 \\
\hline 22 & 41 & $\mathrm{~F}$ & $\mathrm{R}$ & 8 & Yes & 1.0 & 244 & 1.0 & 139 & 12 \\
\hline 23 & 47 & $\mathrm{M}$ & $\mathrm{L}$ & 6 & No & 0.4 & 507 & 1.0 & 145 & 12 \\
\hline 24 & 29 & $\mathrm{M}$ & $\mathrm{L}$ & 6 & No & 0.8 & 463 & 1.0 & 184 & 12 \\
\hline
\end{tabular}

Abbreviations: BCVA, best-corrected visual acuity; CCSC, chronic central serous chorioretinopathy; CFT, central foveal thickness; PED, pigment epithelial detachment; $\mathrm{R}$ vs $\mathrm{L}$, right $v$ s left.

(Table 1). The mean age \pm SD of the patients was $43.7 \pm 8.6$ years (range, $27-61$ years). Seventeen patients were male $(70.8 \%)$ and seven were female $(29.2 \%)$. Three patients (two male and one female) had bilateral CCSC (12.5\%) and the other 21 patients had unilateral CCSC. The primary complaints of patients were central scotoma $(n=9,33.3 \%)$, reduced vision $(n=8,29.6 \%)$, metamorphopsia $(n=6,22.2 \%)$, and micropsia $(n=4,14.8 \%)$.

The mean duration of the current episode was 11.6 \pm 5.9 months (range, 6-24 months). Ten of the eyes had more than one previous episode $(37 \%)$, and the mean number of episodes was $1.37 \pm 0.49$. Eight eyes had combined SRF and PED (29.6\%). The mean PDT laser spot size was $2715.93 \pm 1050.85 \mu \mathrm{m}$ (range, 1350-4500 $\mu \mathrm{m})$.

The BCVA (logMAR) was $0.19 \pm 0.27 \log$ MAR at baseline, $0.10 \pm 0.20 \log$ MAR at 1st month, $0.09 \pm 0.20$ $\log$ MAR at 3rd month, $0.08 \pm 0.20$ at 6 th month, $0.07 \pm 0.20$ at 12 th month, $0.09 \pm 0.25$ at 24 th month, and $0.07 \pm 20$ at the last follow-up. Post-PDT BCVA were all significantly improved compared with the baseline
BCVA $(P<0.001)$ (Figure 1a). The CFT was $329.7 \pm 102.1 \mu \mathrm{m}$ at baseline, $167.5 \pm 23.1 \mu \mathrm{m}$ at $1 \mathrm{st}$ month, $167.0 \pm 23.5 \mu \mathrm{m}$ at 3st month, $162.5 \pm 19.8 \mu \mathrm{m}$ at 6 th month, $170.1 \pm 42.6 \mu$ at 12 th month, $168.0 \pm 17.4 \mu \mathrm{m}$ at 24th month, and $165.4 \pm 21.0 \mu \mathrm{m}$ at the last follow-up. All the measurements of the CFT post-PDT were significantly thinner than the baseline measurement $(P<0.001)$ (Figure 1b). Complete resolution of SRF at the macula was achieved in all eyes at 1 st month and subsequent follow-up visits, except in two eyes, which recurred in the 24th and 36th month (Figure 2).

At the site of the macular detachment, the outer photoreceptor layer (OPL) appeared preserved in 25 eyes, and atrophic in two eyes with symptoms for longer than 24 months. The preserved OPL had an even profile in five eyes and granulated profile in 20 eyes. Granular deposits on RPE also were seen opposite the granulated OPL, as irregularities on RPE (Figure 2).

At baseline, the finding on ICGA was mild-tomoderate hyperfluorescence in all of the eyes. Post-PDT FA and ICGA results at 3rd month demonstrated in all 

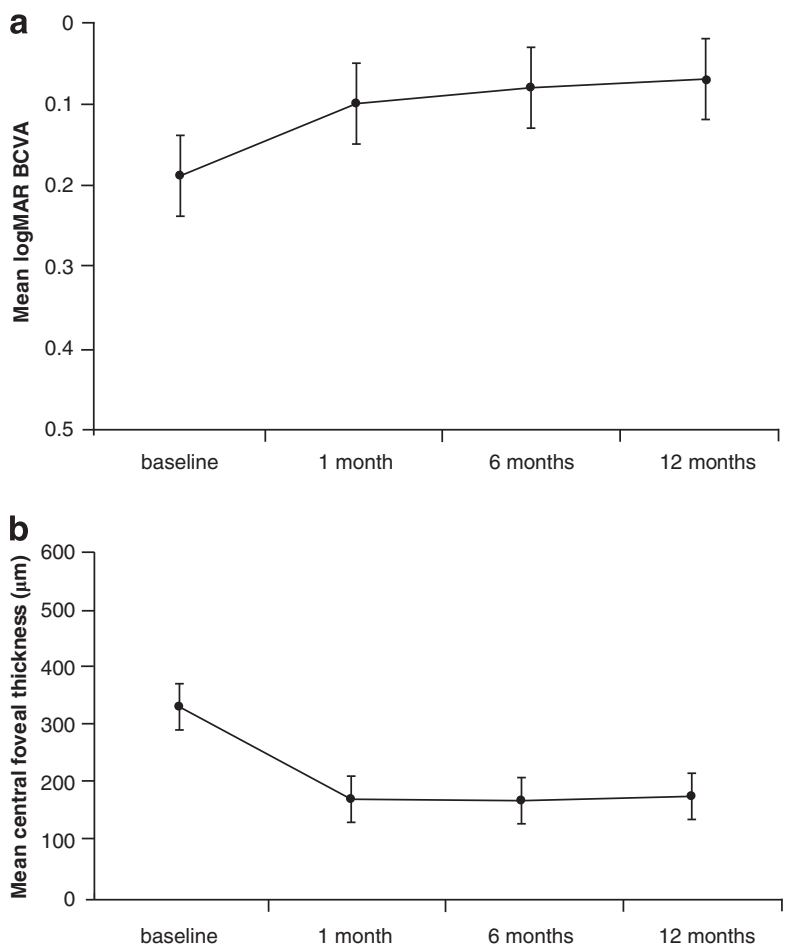

Figure 1 (a) The graph shows the serial changes in mean logarithm of the minimum angle of resolution (logMAR) BCVA of patients. Error bars represent SE of the mean. (b) The graph shows the serial changes in mean OCT central foveal thickness. Error bars represent SE of the mean.

cases complete resolution of choroidal vascular hyperpermeability (Figure 2).

Two eyes had a recurrence at 24th and 36th month, respectively. The patients complained of decreased visual acuity. Recurrent SRF and increased CFT measurements (319 and $354 \mu \mathrm{m}$, respectively) were seen on the OCT. New sites of leakage were seen on the repeated angiography. These two eyes required a second PDT session, and SRF completely resolved a few months later (Figure 3).

FACT was performed at baseline and at 12th month after PDT with best correction under photopic and mesopic conditions, and mesopic condition with glare conditions at five different spatial frequency channels (A: 1.5 cpd, B: 3 cpd, C: 6 cpd, D: 12 cpd, and E: 18 cpd) and displayed in Figure 4. There was significant difference between preop and postop values at all five different spatial frequency channels $(P<0.01)$.

Patients were divided into two groups according to duration of symptoms: 'duration of 6-9 months' and '>9 months' to understand the prognostic effect of duration of symptoms. There were 13 eyes in the first group, and 14 eyes in the second. The differences between the two groups were evaluated in terms of pre- and post-PDT logMAR BCVA, CFT, and contrast sensitivities. For all the mean CFT measurements and contrast sensitivities, no significant difference was found between the two groups $(P>0.05)$. The improvement of BCVA at the 1st, 3rd and 6th months were significantly higher in the first group compared with the second group $(P<0.05)$. However, there was no significant difference of BCVA at 1st year and later between the two groups $(P>0.05)$.

When the 8 eyes with combined SRF and PED were compared with the 19 eyes with only SRF, the improvement of BCVA, CFT and contrast sensitivities did not differ significantly between the two groups $(P>0.05)$.

To evaluate the prognostic effect of age, the patients divided into two groups: ' $<45$ years' was group 1 , and ' $\geq 45$ years' was group 2 . There were 14 patients in the first group, and 10 in the second. BCVA, CFT and contrast sensitivities at baseline and post-PDT follow-up period did not differ significantly between groups $(P>0.05)$.

The mean follow-up period was $25.33 \pm 11.08$ months (range, 12-42 months). The mean BCVA at last follow-up visit was $0.07 \pm 0.20 \log$ MAR. There were no systemic or ocular complications in any of the patients during the follow-up period. All cases had complete anatomic resolution in OCT from the 1st month, and absence of leakage and complete resolution of hyperpermeability on FA and ICGA from the 3th month after treatment until the last visit. The complaints ended in 20 patients after the treatment $(74.1 \%)$, were reduced in 6 patients $(22.1 \%)$, and in only one patient, metamorphopsia persisted while macula was reattached $(3.7 \%)$.

\section{Discussion}

Although CSC usually is a self-limiting and benign condition, some patients may experience persistent chronic macular serous detachment. ${ }^{3-5}$ Recently, PDT with a standard dose of verteporfin has been reported as a useful technique in the treatment of CCSC. ${ }^{11-14}$

ICGA is used to confirm the diagnosis of CCSC, to rule out polypoidal CNV masquerading as CSC, and to provide guidance for the PDT therapy. Yangzi et al ${ }^{11}$ applied ICGA-guided PDT in 20 eyes with CCSC, and treatment led to complete resolution of macular detachment in 12 eyes (60\%), with stable or improved vision in all eyes, although 7 of their 15 patients had a history of photocoagulation. Carrillo Pecorino et al ${ }^{12}$ also performed ICGA-guided PDT for CCSC with a complete resolution of macular serous detachment in $75 \%$ of 16 eyes at 1 month after PDT, and reported that visual improvement of 1 or more lines in $69 \%$ of eyes at 3rd month. Shinojima et $a l^{23}$ believed that if hyperpermeability is not seen on ICGA, PDT would be ineffective. Inoue et $a l^{24}$ also believed that the PDT success rate depends on the degree of hyperpermeability 


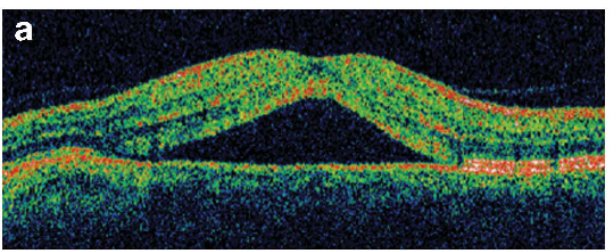

b

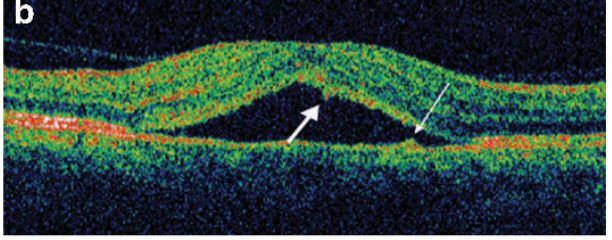

c

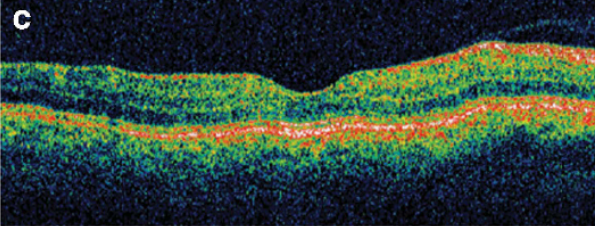

d

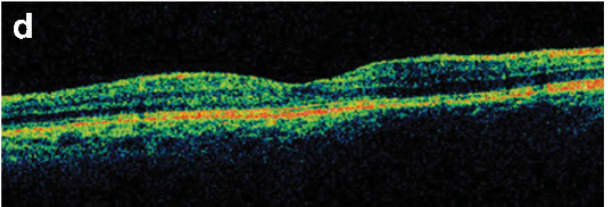

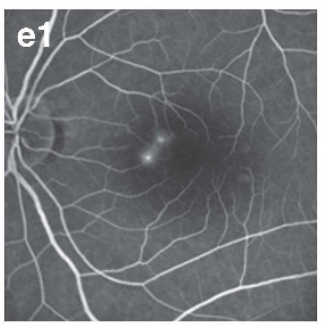
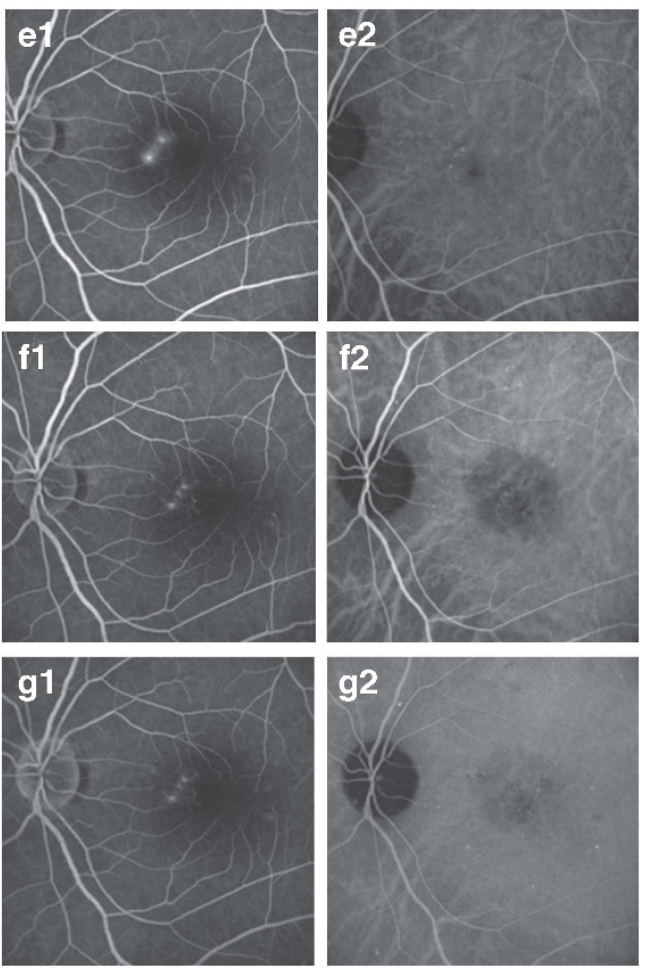

Figure 2 The OCT image (a) shows SRF at diagnosis of acute CSC. The OCT image of same patients at 6th month (b) with granulation of the posterior surface of the detached retina (thick arrow) and granular deposits on RPE (thin arrow). The OCT image of same patient shows the complete resolution of SRF at the macula at 1st month (c) and 24th month (d) after the PDT. The fluorescein angiogram (FA) before PDT shows the leakage from two focus (e1). The iICGA before PDT shows a zone of choroidal hyperpermeability or staining in the submacular area (e2). FA performed 3 months (f1) and 24 months (g1) after the treatment shows no leakage but the window defect in macular area. ICGA image 3 months (f2) and 24 months (g2) after the treatment shows a zone of choroidal hypopermeability (f2), hypopermeable dots and resolution of staining and hyperpermeability (g2).

on ICGA. PDT is not effective or the recurrence rate is predicted to be high in eyes without intense hyperfluorescence. In all our cases, ICGA was used to confirm the diagnosis and guide the laser therapy. At baseline, the finding on ICGA was mild-to-moderate hyperfluorescence in all of the eyes. ICGA-guided treatment of CCSC was directed at the areas of choroidal hyperpermeability. In all cases, as seen in ICGA at the 3rd month, hyperfluorescence decreased significantly after treatment. In this current study, half-dose PDT was performed on 27 eyes of 24 patients diagnosed with CCSC. Treatment resulted in the complete resolution of SRF in all the eyes with improvement in BCVA from the first month. The mean logMAR BCVA of patients at last visit was significantly better compared with the value before treatment.

Potential adverse effects may develop after conventional PDT in CCSC such as RPE atrophy, choroidal ischemia, and secondary CNV. ${ }^{12,15,16}$ Cardillo Piccolino et $\mathrm{al}^{12}$ reported RPE changes in $31 \%$ of eyes, and were thought to be the result of hypoxic damage caused by choriocapillaris occlusion. The development of secondary CNV in one of their six patients series (16.6\%), 3 months after PDT for CCSC has also been reported by Chan et al. ${ }^{15}$ In our study, CNV was not detected in any patients during the follow-up period. The studies demonstrated that PDT was effective in treating CCSC while minimizing the potential adverse effects by reducing the dosage of verteporfin or shortening the time of laser emission, and shortening the interval between infusion and laser application. ${ }^{17-20}$ However, the optimal dosage of verteporfin required to treat CCSC has not been established. To find the safe, effective lowest dose, the effect of half-dose verteporfin was compared with one-third-dose verteporfin PDT, and half-dose PDT was observed to be more effective in the resolution of SRF for CCSC. $^{25}$

Prolonged detachment of the macula is known to lead to irreversible structural changes within the neurosensory retina, limiting the visual recovery 

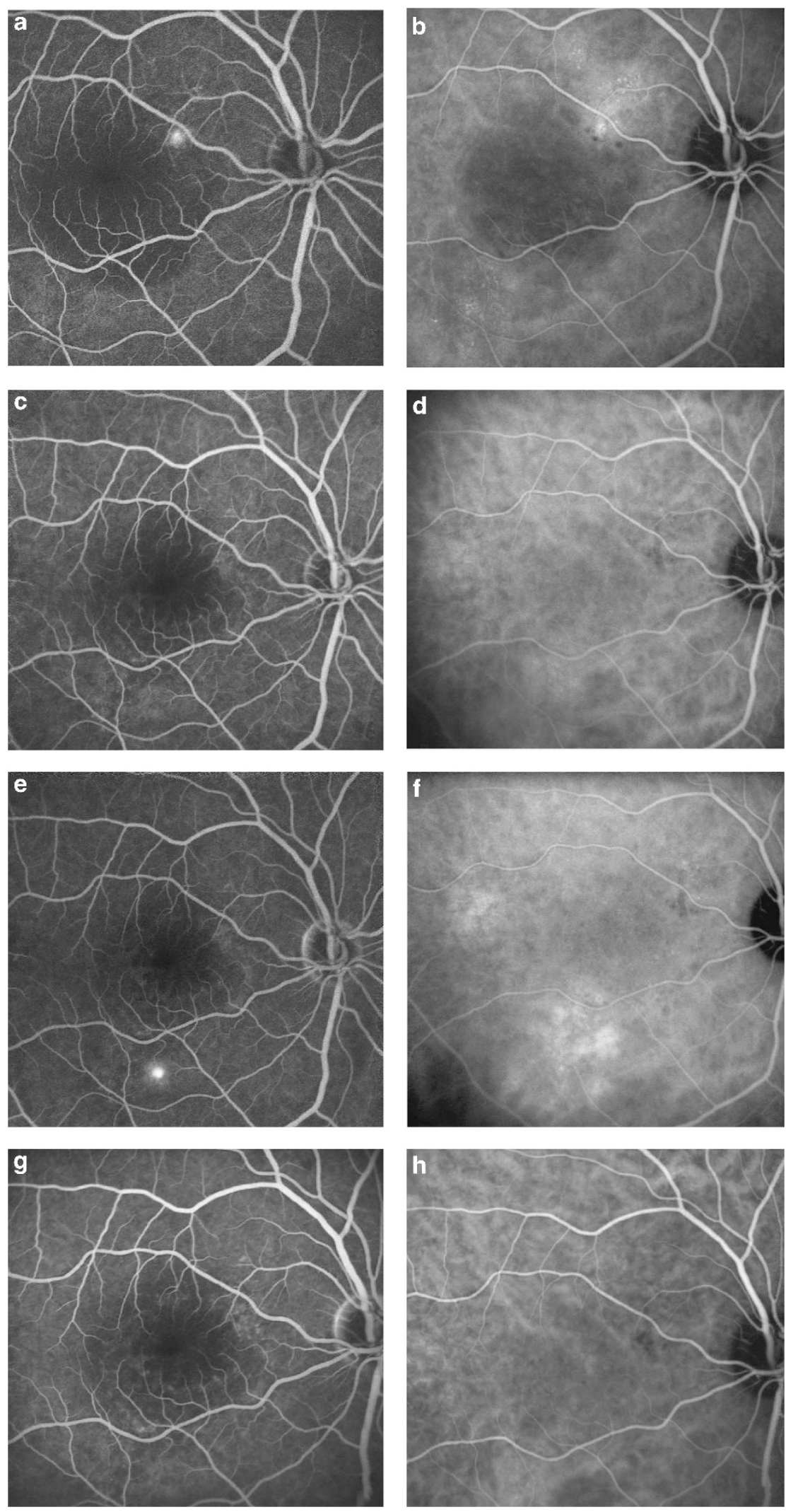
potential. ${ }^{11,26}$ At the site of the macular detachment, Piccolino et $a l^{26}$ observed a preserved photoreceptor layer with an even or granulated outer profile or an atrophic photoreceptor layer. A preserved undamaged OPL becomes thickened and granulated after several months of detachment and then progressively thins when detachment persist for years. After the macular detachment resolved, they observed a significantly better VA in eyes with a preserved OPL than in eyes with an atrophic OPL. In our study, we observed only two eyes with an atrophic OPL, and these eyes were symptomatic for at least 2 years. At baseline, BCVA of these eyes were lower than the others, and poor VA improvement was observed after foveal reattachment.

Moreover, poor vision at the beginning and prolonged serous macular detachment seem to be poor prognostic factors. ${ }^{11,26}$ Just because of this, patients were evaluated in two groups according to the duration of symptoms: 'duration of 6-9 months' and '>9 months', to understand the prognostic effect of duration of symptoms. The improvement of BCVA at the 1st, $3 \mathrm{rd}$ and 6th months were found to be significantly higher in the first group compared with that in the second group $(P<0.05)$, but at 1 st year and later, there was no significant difference between the two groups $(P>0.05)$. This means that shortened duration of macular detachment may cause better or quicker response to treatment as functional result, and early treatment is an influencing factor for visual improvement. ${ }^{11,26}$ Moreover, because many patients are of working age, visual symptoms may interfere with their activities considerably. The treatment would be able to shorten the duration of symptoms. Based on all of this, the treatment may be shifted to an earlier time depending on the tendency to become chronic or for some individual factors for better functional results.

The eyes with combined SRF and PED were compared with the eyes with only SRF; the improvement of BCVA, CFT and contrast sensitivities did not differ significantly between the two groups $(P>0.05)$. As the location of PED was extrafoveal in most of our cases (six of eight), extrafoveal atrophy due to PED did not affect central vision significantly. Mudvari et $a l^{27}$ reported that PED in
CSC cases generally has a good visual prognosis, confirming previous reports. The most frequent outcome is resolution of the PED with subsequent pigmentary atrophy. Persistence of the PED occurs less frequently. Subfoveal PED is uncommon and may be associated with a poorer visual prognosis. In our series, in all cases, PED and SRF resolved completely, only in two eyes recurrence from new foci were seen 24 and 36 months after treatment. In one of them, PED was present.

To evaluate retinal functional changes after half-dose PDT, multifocal electroretinogram (mfERG) or MP-1 microperimetry used by authors in previous studies. The changes in BCVA and OCT CFT findings also correlated with mfERG responses. The results showed a significant improvement in the macular sensitivity after half-dose PDT. ${ }^{28-31}$ In our study, we used FACT to evaluate the macular function, because it has been reported that both visual acuity and contrast sensitivity are strongly associated with the ability to perform vision-related activities. The improvement of contrast sensitivities was significant after foveal reattachment $(P<0.01)$.

These long-term results showed us that the use of half-dose verteporfin seemed to be safe and effective. The development of secondary CNV or severe RPE atrophy in the treated patients was not observed. The recurrence of CCSC was observed only in two eyes and these eyes were re-treated successfully with half-dose PDT.

One of the limitations of this study is the lack of control groups of conventional PDT or a placebo group. Further prospective studies with control groups are needed to determine the effective lowest dose of verteporfin. In addition to reducing the dose of verteporfin, the functional and anatomic effects of reducing other variables such as laser power and duration of laser application should also be evaluated in further studies. Additional evaluation is required to determine the optimal timing of treatment for the preservation of macular function. Further randomized placebocontrolled studies with functional evaluation methods such as FACT can help to determine the effective lowest dose of verteporfin or laser power, and the optimal timing of treatment.

Figure 3 The images regarding one of the two cases that recurred. The fluorescein angiogram (FA) before PDT (a) shows the focal leakage. The ICGA before PDT (b) shows choroidal hyperpermeability and staining. A hypofluorescent area caused by subretinal fluid can be seen on FA and ICGA (a,b). FA performed 3 months after the PDT (c) shows no leakage but the window defect. ICGA 3 months after the treatment (d) shows hypopermeable dots and resolution of staining and hyperpermeability. After 24 months, the patient complained of decreased vision on the same eye. New focus of leakage were seen on the repeated FA (e). Repeated ICGA (f) shows hyperfluorescence secondary to choroidal hyperpermeability and staining on the other site of macula. A second half-dose PDT session performed on the area of choroidal hyperpermeability. FA performed 3 months after the second PDT (g) shows no leakage but the window defects in macular area. ICGA 3 months after the second treatment (h) shows hypopermeable dots and resolution of staining and hyperpermeability. 
a

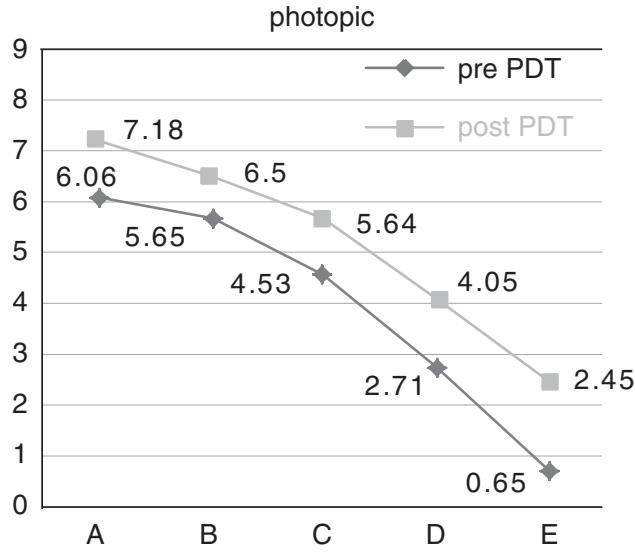

b

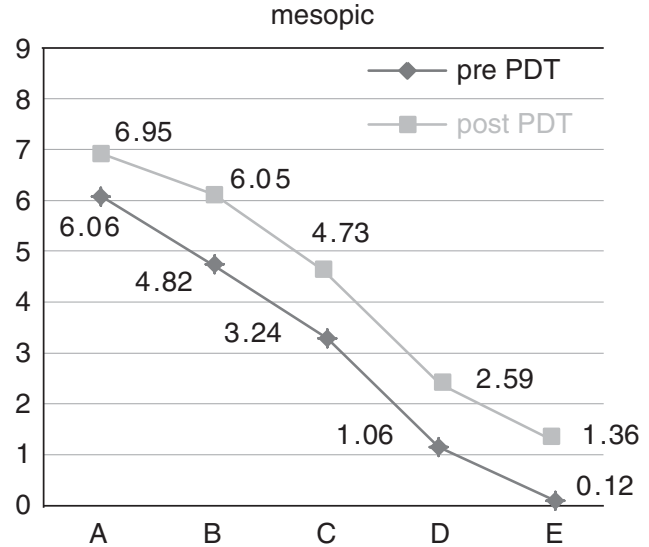

C

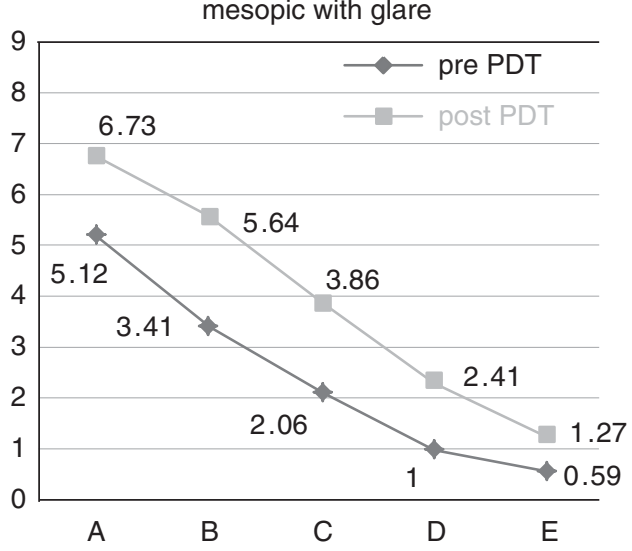

Figure 4 FACT was performed at baseline and 12th months post PDT under photopic (a) and mesopic (b) conditions, and mesopic condition with glare (c) conditions at five different spatial frequency channels (A: 1.5 cycle per degree (cpd), B: 3 cpd, C: $6 \mathrm{cpd}, \mathrm{D}: 12 \mathrm{cpd}$, and E: $18 \mathrm{cpd}$ ).

In conclusion, PDT with half-dose verteporfin is an effective and safe treatment for CCSC as demonstrated in the long-term results. In addition to visual and anatomic improvement, measurement of contrast sensitivity by FACT can be useful for the evaluation of functional effectiveness of half-dose PDT for CCSC.

\section{Summary}

\section{What was known before}

- Photodynamic therapy is an option for the treatment of chronic central serous chorioretinopathy (CCSC).

\section{What this study adds}

- Half-dose photodynamic therapy is an effective and safe method in the treatment of CCSC with anatomical and functional success as long-term results (up to 3 years) of this study.

\section{Conflict of interest}

The authors declare no conflict of interest.

\section{References}

1 Gass JD. Pathogenesis of disciform detachment of neuroepithelium. Am J Ophthalmol 1967; 63: 587-615.

2 Yannuzzi LA. Type-A behaviour and central serous chorioretinopathy. Retina 1987; 7: 111-131.

3 Yannuzzi LA, Gitter KA, Schatz H. Central serous chorioretinopathy. In: The macula: A Comprehensive Text and Atlas. Williams and Wilkins: Baltimore, USA; 1979. pp 145-165.

4 Yannuzzi LA, Shakin JL, Fisher YL, Altomonte MA. Peripheral retinal detachments and retinal pigment epithelial atrophic tracts secondary to central serous pigment epitheliopathy. Ophthalmology 1984; 91: 1554-1572.

5 Spaide RF, Campeas L, Haas A, Yannuzzi LA, Fisher YL, Guyer DR et al. Central serous chorioretinopathy in older and younger adults. Ophthalmology 1996; 103: 2070-2079.

6 Guyer DR, Yannuzzi LA, Slakter JS, Sorenson JA, Hope-Ross M, Orlock DR. Digital indocyanine green videoangiography of central serous chorioretinopathy. Arch Ophthalmol 1994; 112: 1057-1062.

7 Prunte C, Flammer J. Choroidal capillary and venous congestion in central serous chorioretinopathy. Am J Ophthalmol 1996; 121: 26-34.

8 Scheider A, Nasemann JE, Lund OE. Fluorescein and indocyanine green angiographies of central serous choroidopathy by scanning laser ophthalmoscopy. Am J Ophthalmol 1993; 115: 50-56.

9 Piccolino FC, Borgia L. Central serous chorioretinopathy and indocyanine green angiography. Retina 1994; 14: 231-242.

10 Spaide RF, Hall H, Haas A, Campeas L, Yannuzzi LA, Fisher YL et al. Indocyanine green videoangiography of older patients with central serous chorioretinopathy. Retina 1996; 16: 203-213.

11 Yannuzzi LA, Slakter JS, Gross NE, Spaide RF, Costa DL, Huang SJ et al. Indocyanine green angiography-guided photodynamic therapy for treatment of chronic central serous chorioretinopathy: a pilot study. Retina 2003; 23: 288-298.

12 Cardillo Piccolino F, Eandi CM, Ventre L, Rigault de la Longrais RC, Grignolo FM. Photodynamic therapy for chronic central serous chorioretinopathy. Retina 2003; 23: 752-763.

13 Shanmugam PM, Agarwal M. Indocyanine green angiography-guided photodynamic therapy for treatment 
of chronic central serous chorioretinopathy: a pilot study. Retina 2004; 24: 988-989.

14 Taban M, Boyer DS, Thomas EL, Taban M. Chronic central serous chorioretinopathy: photodynamic therapy. Am J Ophthalmol 2004; 137: 1073-1080.

15 Chan WM, Lam DS, Lai TY, Tam BS, Liu DT, Chan CK. Choroidal vascular remodelling in central serous chorioretinopathy after indocyanine green guided photodynamic therapy with verteporfin: a novel treatment at the primary disease level. Br J Ophthalmol 2003; 87: 1453-1458.

16 Colucciello M. Choroidal neovascularization complicating photodynamic therapy for central serous retinopathy. Retina 2006; 26: 239-242.

17 Lai TY, Chan WM, Li H, Lai RY, Liu DT, Lam DS. Safety enhanced photodynamic therapy with half-dose verteporfin for chronic central serous chorioretinopathy: a short term pilot study. Br J Ophthalmol 2006; 90: 869-874.

18 Chan WM, Lai TY, Lai RY, Tang EW, Liu DT, Lam DS. Safety enhanced photodynamic therapy for chronic central serous chorioretinopathy: one-year results of a prospective study. Retina 2008; 28(1): 85-93.

19 Lim JW, Kang SW, Kim YT, Chung SE, Lee SW. Comparative study of patients with central serous chorioretinopathy undergoing focal laser photocoagulation or photodynamic therapy. Br J Ophthalmol 2011; 95(4): 514-517.

20 Jirarattanasopa P, Ratanasukon M, Bhurayanontachai P. The one-year results of half-dose photodynamic therapy with verteporfin in chronic or recurrent central serous chorioretinopathy. J Med Assoc Thai 2012; 95(Suppl 4): S56-S60.

21 Nicolò M, Zoli D, Musolino M, Traverso CE. Association between the efficacy of half-dose photodynamic therapy with indocyanine green angiography and optical coherence tomography findings in the treatment of central serous chorioretinopathy. Am J Ophthalmol 2012; 153(3): 474-480.

22 Ginsburg AP. Clinical findings from a new contrast sensitivity test chart. In: Fiorentini A, Guyton DL, Siegel IM (eds). Advances in Diagnostic Visual Optics. Springer-Verlag: Berlin, Germany, 1987, pp 132-145.
23 Shinojima A, Kawamura A, Mori R, Fujita K, Yuzawa M. Detection of morphologic alterations by spectral-domain optical coherence tomography before and after half-dose verteporfin photodynamic therapy in chronic central serous chorioretinopathy. Retina 2011; 31(9): 1912-1920.

24 Inoue R, Sawa M, Tsujikawa M, Gomi F. Association between the efficacy of photodynamic therapy and indocyanine green angiography findings for central serous chorioretinopathy. Am J Ophthalmol 2010; 149(3): 441-446.

25 Uetani R, Ito Y, Oiwa K, Ishikawa K, Terasaki H. Half-dose vs one-third-dose photodynamic therapy for chronic central serous chorioretinopathy. Eye 2012; 26(5): 640-649.

26 Piccolino FC, de la Longrais RR, Ravera G, Eandi CM, Ventre L, Abdollahi A et al. The foveal photoreceptor layer and visual acuity loss in central serous chorioretinopathy. Am J Ophthalmol 2005; 139(1): 87-99.

27 Mudvari SS, Goff MJ, Fu AD, McDonald HR, Johnson RN, Ai E et al. The natural history of pigment epithelial detachment associated with central serous chorioretinopathy. Retina 2007; 27(9): 1168-1173.

28 Wu ZH, Lai RY, Yip YW, Chan WM, Lam DS, Lai TY. Improvement in multifocal electroretinography after halfdose verteporfin photodynamic therapy for central serous chorioretinopathy: a randomized placebo-controlled trial. Retina 2011; 31(7): 1378-1386.

29 Fujita K, Yuzawa M, Mori R. Retinal sensitivity after photodynamic therapy with half-dose verteporfin for chronic central serous chorioretinopathy: short-term results. Retina 2011; 31(4): 772-778.

30 Senturk F, Karacorlu M, Ozdemir H, Karacorlu SA, Uysal O. Microperimetric changes after photodynamic therapy for central serous chorioretinopathy. Am J Ophthalmol 2011; 151(2): 303-309.

31 Fujita K, Shinoda K, Imamura Y, Matsumoto CS, Mizutani Y, Mizota A et al. Correlation of integrity of cone outer segment tips line with retinal sensitivity after half-dose photodynamic therapy for chronic central serous chorioretinopathy. Am J Ophthalmol 2012; 154(3): 579-585. 